Objectives Rheumatoid arthritis (RA) is characterised by chronic synovial inflammation, which results in progressive polyarticular destruction. Immunohistological characterisation of infiltrating cells may help to elucidate the role of specific chemokines and their receptors in pathological processes. In this regard we previously described high expression of chemokine receptor CXCR-3 in synovial tissue. Now we have investigated expression of chemotactic factors IP-10 and Mig, which are ligands for CXCR-3. Methods Synovial tissue samples were obtained from 5 RA patients who have undergone total joint replacement or synovectomy. Native cryostat tissue sections were examined for CD3, CD4, CD8, CD19, CD14, CD31, CD68, CXCR-3, IP-10 and Mig with mouse anti-human monoclonal antibodies. Two-step visualisation with DAKO EnVision was applied. This visualisation system is based on enzyme-conjugated dextrane polymer, which carriers secondary antibody molecules. Negative controls were performed by using izotype-matched irrelevant antibodies. Results A strong positive reaction for CXCR-3 was detected on lymphocytes and macrophage-like synoviocytes; weak and irregular staining was seen in endothelial cells. IP-10 and Mig were expressed in all tissue samples. The positivity was found mainly in the lymphoid infiltrating cells and the staining also co-localised with CD68 positive synoviocytes. In addition, a stronger expression of IP-10 than of Mig was seen, particularly in tissues with a strong vascularity. Endothelial cells were strongly positive for IP-10 and weakly also for Mig. In general, IP-10 and Mig staining was observed in sporadic aggregates surrounding blood vessels.

Conclusion High expression of IP-10 and Mig on a majority of the infiltrating cells suggests a role for interferon-g in RA inflammation, as IP-10 and Mig are induced by this cytokine. They may play a role in the attraction of CD4 positive T cells into the synovial environment. Furthermore, a frequent expression of CXCR3 on synovial resident cells propounds a possible role for IP-10 and Mig in their activation, perhaps even in the autocrine fashion. This work was supported by a grant NI/6459 from IGA MZ CR.

\section{THU0107 METHOTREXATE COVALENTLY COUPLED TO HUMAN SERUM ALBUMIN (MTX-HSA) TARGETS TO INFLAMED JOINTS AND IS SUPERIOR TO METHOTREXATE INHIBITING THE DEVELOPMENT OF MURINE COLLAGEN- INDUCED ARTHRITIS (CIA)}

${ }^{1} \mathrm{~A}$ Wunder, ${ }^{2}$ EH Stelzer, ${ }^{1} \mathrm{H}$ Sinn, ${ }^{3} \mathrm{~J}$ Funk, ${ }^{3} \mathrm{AD}$ Ho, ${ }^{3} \mathrm{C}$ Fiehn. ${ }^{1}$ Department E0300, German Cancer Research Center; ${ }^{2}$ Light Microsc. Gr., Europ. Lab. for Mol. Biol; ${ }^{3}$ Department of Internal Medicine V, University of Heidelberg, Heidelberg, Germany

\subsection{6/annrheumdis-2001.984}

Background Methotrexate (MTX) is a well established drug for the treatment of rheumatoid arthritis (RA). Pharmacological studies have shown, that most of the drug is eliminated by the kidneys. This results in a short plasma half-life and low concentrations of MTX in inflamed joints. In studies with tumour-bearing rats we showed, that due to the pathophysiological conditions in solid tumours and the high demand of tumour cells for amino acids and energy, albumin accumulates in tumours (up to $20 \%$ of the initially injected amount) and only a relatively small proportion is found in the liver (about 6\%). Covalent coupling of MTX, fluorescent dyes and radioactive labels in a 1:1 molar ratio to albumin leads to conjugates which behave in-vivo like native albumin. In phase I clinical trials with tumour patients MTX-HSA showed promising results and relatively high dosages were well tolerated over several years. Like tumour cells, synovial cells of patients with RA show a high rate of proliferation and are in an active metabolic state. The plasma protein albumin might therefore be an appropriate carrier to increase drug concentrations in inflamed joints.

Objectives To proof if albumin is a suitable drug carrier to target MTX to inflamed joints in an RA animal model and isolated human RA synoviocytes.

Methods Laseroptic, scintigraphic and radiopharmacokinetic experiments with fluorescent and radioactive labelled albumin were performed to study the distribution of albumin in mice with and without CIA ( $\mathrm{n}=5$ each point of the time course). The uptake of fluorescence labelled albumin by human RA synoviocytes was investigated by laser scanning microscopy in-vitro. The pharmacokinetics of MTX in arthritic mice was studied using 3H-labelled MTX. The therapeutic efficiency of MTXHSA, MTX and a combination of both drugs (injected in different dosages $2 \times /$ week) was investigated by its ability to inhibit the development of arthritis ( $n=3-35 /$ group).

Results About 3\% of the initially injected radioactive albumin was detected in each inflamed paw within a few hours after application, while in healthy paws only $0.3 \%$ of the tracer was found (accumulation by a factor of 6 in respect to the weight of the paws). Scintigraphic scans confirmed this results. Only $0.03 \%$ of $3 \mathrm{H}-\mathrm{MTX}$ was measured in the inflamed paws. In the liver only $7 \%$ of the radiolabeled albumin but $27 \%$ of $3 \mathrm{H}-\mathrm{MTX}$ was found. Illumination by laser light after injection of fluorescent labelled albumin showed strong fluorescence in arthritic but not in healthy joints of paws affected by CIA. The examination of human synoviocytes in-vitro showed catabolism of albumin in lysosomes. In equivalent dosages (7.5 mg MTX/kg twice a week) MTX-HSA was significantly $(\mathrm{p}=0.017)$ superior to MTX in inhibiting the development of CIA (control: 68\% arthritis incidence, MTX: 57\%, MTX-HSA 28\%). To achieve a comparable result as MTX-HSA almost a five times higher dosage of MTX (35 mg/kg) was necessary (37\% arthr. inc.). Both drugs act synergistically $(3.75 \mathrm{mg} / \mathrm{kg}$ MTX plus $0.8 \mathrm{mg} / \mathrm{kg}$ MTX-HSA: $29 \%$ arthr. inc.) in respect that $1.6 \mathrm{mg} / \mathrm{kg}$ MTX-HSA had no significant effect (57\%).

Conclusion Using albumin as drug carrier allows to target drugs such as MTX specifically to inflamed joints. This might increase the therapeutic efficacy of MTX in human RA. Clinical trials to proof this concept are in preparation.

\section{THU0108 ANTIBODIES TO PARVOVIRUS B19 IN RHEUMATOID ARTHRITIS AND OSTEOARTHRITIS}

${ }^{1} \mathrm{P}$ Athanassiou, ${ }^{2} \mathrm{~K}$ Themeli-Digalaki, ${ }^{1} \mathrm{~A}$ Elezoglou, ${ }^{2} \mathrm{E}$ Orkopoulou, ${ }^{1} \mathrm{~A}$ Theodorou, ${ }^{1} \mathrm{R}$ Karidis, ${ }^{2} \mathrm{C}$ Koutsia-Karouzou, ${ }^{1} \mathrm{G}$ Vezyroglou. ${ }^{1}$ Department of Rheumatology; ${ }^{2}$ Department of Clinical Microbiology, Asklepieion Hospital, Voula, Athens, Greece

\subsection{6/annrheumdis-2001.985}

Background Parvovirus B19 may cause an acute or chronic polyarthropathy, which in many cases fulfils the criteria of the American College of Rheumatology for the diagnosis of rheumatoid arthritis (RA). Additionally, parvovirus B19 has been implicated in the pathogenesis of RA.

Objectives The aim was to investigate the presence of antibodies to parvovirus B19 in patients with RA and osteoarthritis (OA).

Methods In the study were included 50 patients with RA, 24 patients with $\mathrm{OA}$ and 26 healthy controls. In all patients IgG and IgM antibodies to parvovirus B19 were measured by indirect immunofluorescence. In patients found to be positive for IgM 